\title{
Transcranial magnetic stimulation therapeutic applications on sleep and insomnia: a review
}

\author{
Ricardo Oroz ${ }^{1}$, Simon Kung ${ }^{2}$, Paul E. Croarkin ${ }^{2}$ and Joseph Cheung ${ }^{3 *}$ (D)
}

\begin{abstract}
Repetitive transcranial magnetic stimulation (rTMS) is a neuromodulatory technique approved by the US Food and Drug Administration for use in treatment-resistant major depressive disorder. It works by generating localized magnetic fields that create depolarizing electrical currents in neurons a few centimeters below the scalp. This localized effect is believed to stimulate neural plasticity, activate compensatory processes, and influence cortical excitability. Additionally, rTMS has been used in a variety of clinical trials for neurological and psychiatric conditions such as anxiety, post-traumatic stress disorder and epilepsy. Beneficial effects in sleep parameters have been documented in these trials, as well as in major depressive disorder, and have led to an interest in using rTMS in the field of sleep medicine for specific disorders such as insomnia, hypersomnia, and restless legs syndrome. It is unknown whether rTMS has intrinsically beneficial properties when applied to primary sleep disorders, or if it only acts on sleep through mood disorders. This narrative review sought to examine available literature regarding the application of rTMS for sleep disorder to identify knowledge gaps and inform future study design. The literature in this area remains scarce, with few randomized clinical trials on rTMS and insomnia. Available studies have found mixed results, with some studies reporting subjective sleep improvement while objective improvement is less consistent. Due to the heterogeneity of results and the variations in rTMS protocols, no definitive conclusions have been reached, signaling the need for further research.
\end{abstract}

Keywords: Transcranial magnetic stimulation, Insomnia, Sleep disorders

\section{Introduction}

Transcranial magnetic stimulation (TMS) is a relatively novel method for the treatment of a variety of neuropsychiatric conditions. This noninvasive procedure generates magnetic fields to create an electrical current to depolarize neurons and modulate neuronal activity in localized cortical regions a few centimeters below the scalp (Valero-cabré et al. 2017). Repetitive TMS (rTMS) is approved by the US Food and Drug Administration for treatment-resistant major depressive disorder (MDD) (Berlim et al. 2013a; Berlim et al. 2014; Fitzgerald et al.

\footnotetext{
* Correspondence: Cheung.joseph@mayo.edu

${ }^{3}$ Division of Pulmonary, Allergy and Sleep Medicine, Mayo Clinic, 4500 San Pablo Rd, Jacksonville, FL 32224, USA

Full list of author information is available at the end of the article
}

2012; Ray et al. 2011; George et al. 2010; O’Reardon et al. 2007) (see Fig. 1) and since 2018 for obsessivecompulsive disorder (Carmi et al. 2019; Carmi et al. 2018; Berlim et al. 2013b). In addition, it has been shown to have potential benefits in multiple other conditions, including anxiety (Rodrigues et al. 2019; Cirillo et al. 2019), posttraumatic stress disorder (Kozel et al. 2018; Watts et al. 2012; Karsen et al. 2014; Ahmadizadeh and Rezaei 2018), and fibromyalgia (Boyer et al. 2014; Passard et al. 2007; Mhalla et al. 2011).

rTMS has an excellent tolerability profile. The most common adverse effects include scalp discomfort/pain, facial muscle twitching, and headache (O'Reardon et al. 2007). Rare adverse effects include seizures, hypomania (Rossi et al. 2009). In general, caution is recommended

(c) The Author(s). 2021, corrected publication 2021. Open Access This article is licensed under a Creative Commons Attribution 4.0 International License, which permits use, sharing, adaptation, distribution and reproduction in any medium or format, as long as you give appropriate credit to the original author(s) and the source, provide a link to the Creative Commons licence, and indicate if changes were made. The images or other third party material in this article are included in the article's Creative Commons licence, unless indicated otherwise in a credit line to the material. If material is not included in the article's Creative Commons licence and your intended use is not permitted by statutory regulation or exceeds the permitted use, you will need to obtain permission directly from the copyright holder. To view a copy of this licence, visit http://creativecommons.org/ licenses/by/4.0/. 


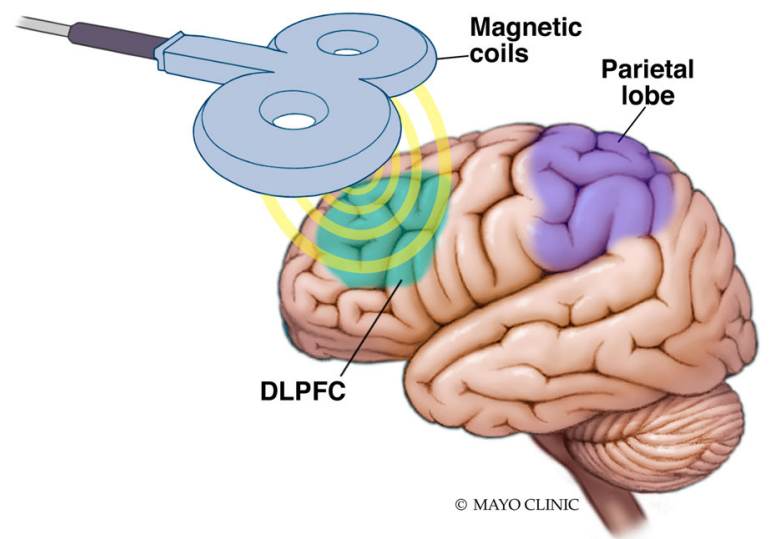

Fig. 1 rTMS -- a noninvasive therapy where magnetic fields are applied on the scalp to create an electrical current in brain tissue to modulate neuronal activity in localized cortical regions

in patients with pacemakers as well as a history of seizures (Valero-cabré et al. 2017; Rossi et al. 2009). Absolute contraindications include metallic material in the head near the machine coil such as cochlear implants (Valero-cabré et al. 2017; Rossi et al. 2009).

Sleep disorders are common in the general population (Ohayon 2011) and in patients suffering from MDD (Ohayon 2011; Murphy and Peterson 2015). Several studies have reported that rTMS for various conditions improved sleep quality by subjective measures (van Dijk et al. 2009; Sánchez-Escandón et al. 2016), even in those who experienced no improvement with the actual mood disorder (Sonmez et al. 2020); hence, it has recently been suggested that rTMS could have an intrinsic positive effect on sleep outside of its application in MDD (Sonmez et al. 2020). Neuroimaging studies have found that patients with chronic primary insomnia often exhibit cortical hyperexcitability (Van Der Werf et al. 2010; Lanza et al. 2015). It was shown that chronic insomnia has specific pathophysiologic features that differ from other sleep disordered states such as intermittent sleep deprivation, with hyperarousal being a core feature (Riemann et al. 2015; Riemann et al. 2010). Notwithstanding, sleep deprivation in healthy individuals has been shown to promote increased cortical excitability (Kreuzer et al. 2011; Civardi et al. 2001).

rTMS can be used as a mapping tool to study neuronal networks for pre-surgical planning, diagnostic purposes (Valero-cabré et al. 2017), and to examine brain cortex functionalities (Valero-cabré et al. 2017; Nardone et al. 2013). In addition, it has recently been explored in neurology and psychiatry communities to determine its therapeutic potential for different sleep pathologies such as insomnia (Feng et al. 2019), sleep bruxism (Zhou et al. 2016), and restless legs syndrome (Lin et al. 2015). To date, the exact mechanism by which rTMS is thought to influence sleep has yet to be fully explained.
It is believed that rTMS engages neuronal plasticity to modulate compensatory brain processes (Valero-cabré et al. 2017), and has been shown to regulate altered cortical states through the dorsolateral prefrontal cortex (DLPFC) in patients with MDD (Berlim et al. 2013a; George et al. 2010). Therefore, in theory, rTMS could benefit patients with sleep pathologies such as insomnia, obstructive sleep apnea, and restless legs syndrome which have been shown to have altered cortical features (Lanza et al. 2015). rTMS also has been found to influence sleep and its effects by, for example, increasing slow-wave activity (Huber et al. 2007) and reducing the impact of sleep deprivation on memory (Luber et al. 2013). In this review, we examine the current scientific understanding of rTMS therapy for use in sleep disorders, with a focus on insomnia, and discuss findings from various studies.

\section{Methods}

We conducted a literature search in PubMed on October 31, 2019, using keywords of "TMS", "transcranial magnetic stimulation", "sleep”, "sleep disorders", and "insomnia." Scopus and Google Scholar databases were also searched but did not yield additional references. Our inclusion criteria were studies regarding therapeutic applications, both openlabel and randomized controlled trials, on sleep pathologies. Studies which employed TMS for diagnosis, neural mapping, pathophysiological analysis or animal studies were excluded. Figure 2 illustrates these criteria and our search results. An example of a search performed in PubMed with $\mathrm{MeSH}$ is: ("Transcranial Magnetic Stimulation" [MeSH]) AND ("Transcranial Magnetic Stimulation/therapeutic use"[MeSH]) AND ("Transcranial Magnetic Stimulation/ therapy" $[\mathrm{MeSH}]$ ) OR ("Dyssomnias"[MeSH]) OR ("Sleep Initiation and Maintenance Disorders" [MeSH]) OR ("Sleep" [MeSH]) OR ("Sleep Disorders, Intrinsic"[MeSH]). We abstracted information about the sample size, stimulation location, TMS parameters (frequency, motor threshold), duration of treatment, concurrent medication use, rating scales used, and subjective and objective outcomes.

\section{Results}

A total of 680 search results were screened with 20 studies being selected for inclusion and analysis. Table 1 summarizes the principal 15 studies, while additional 5 are discussed throughout the review. Out of the principal fifteen studies in Table 1 ten were open-label, and five were randomized. A wide variety of stimulation locations, frequencies, motor thresholds, and duration of treatment were found. For the outcomes, most studies employed a combination of subjective (questionnaires) and objective (PSG, actigraphy, biological) measures. 
PubMed studies categorized by keywords

TMS Insomnia: 15 results

Transcranial magnetic stimulation

insomnia: 28 results

TMS Sleep: 176 results

transcranial magnetic stimulation

sleep: 363 results

TMS sleep disorders: 98 results

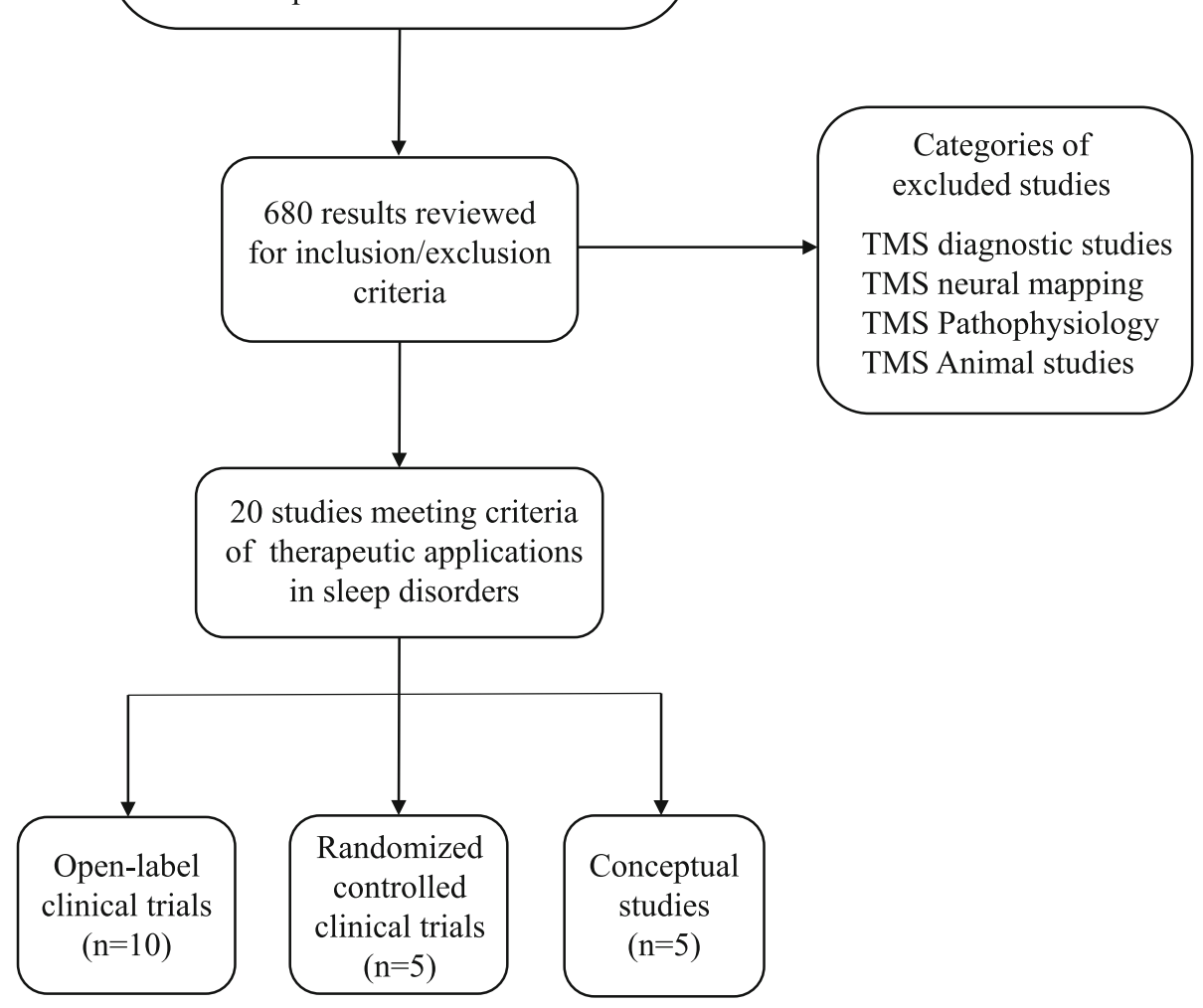

Fig. 2 Flow diagram of reviewed studies

\section{Stimulation frequency and motor threshold}

Broadly, rTMS can be dosed with high and low frequencies. High frequency (HF) is considered to be any frequency above $1 \mathrm{~Hz}$, more typically set at $\sim 10 \mathrm{~Hz}$. HF has been shown to have an excitatory effect on the frontal cortex and is commonly used in MDD (Valero-cabré et al. 2017; Hallett 1910; Aleman 2013; Lefaucheur et al. 2014). On the other hand, low-frequency (LF) rTMS includes frequencies $1 \mathrm{~Hz}$ and below. LF rTMS, when used continuously, is thought to be inhibitory (Valero-cabré et al. 2017; Chen et al. 1997; Romero et al. 2002). Six of the studies used high frequency stimulation, 8 used low frequency, and one used a combination of high and low frequencies.

Resting motor threshold (MT), another important rTMS parameter, is the minimum amount of stimulation needed to create a motor-evoked potential to cause muscle movement. The muscle movement is usually visually observed from a twitch of the hand muscles or the abductor pollicis brevis (thumb) muscle and indicates the required rTMS potency to stimulate neurons in each patient. The included studies used stimulation intensities ranging from 80 to $120 \%$ of MT. For MDD, a stimulation intensity of $120 \%$ of MT is commonly used.

\section{Effect of frequency and location}

A number of neurologic and psychiatric disorders have been found to display characteristic disturbances in cortical excitability. In MDD, diminished activity in the frontal cortex is commonly found (Kimbrell et al. 2002; Mayberg 2003). In contrast, chronic insomnia often shows increased cortical excitability (Van Der Werf et al. 2010; Lanza et al. 2015).

While the importance of rTMS frequency regarding clinical outcomes has been the subject of investigation 


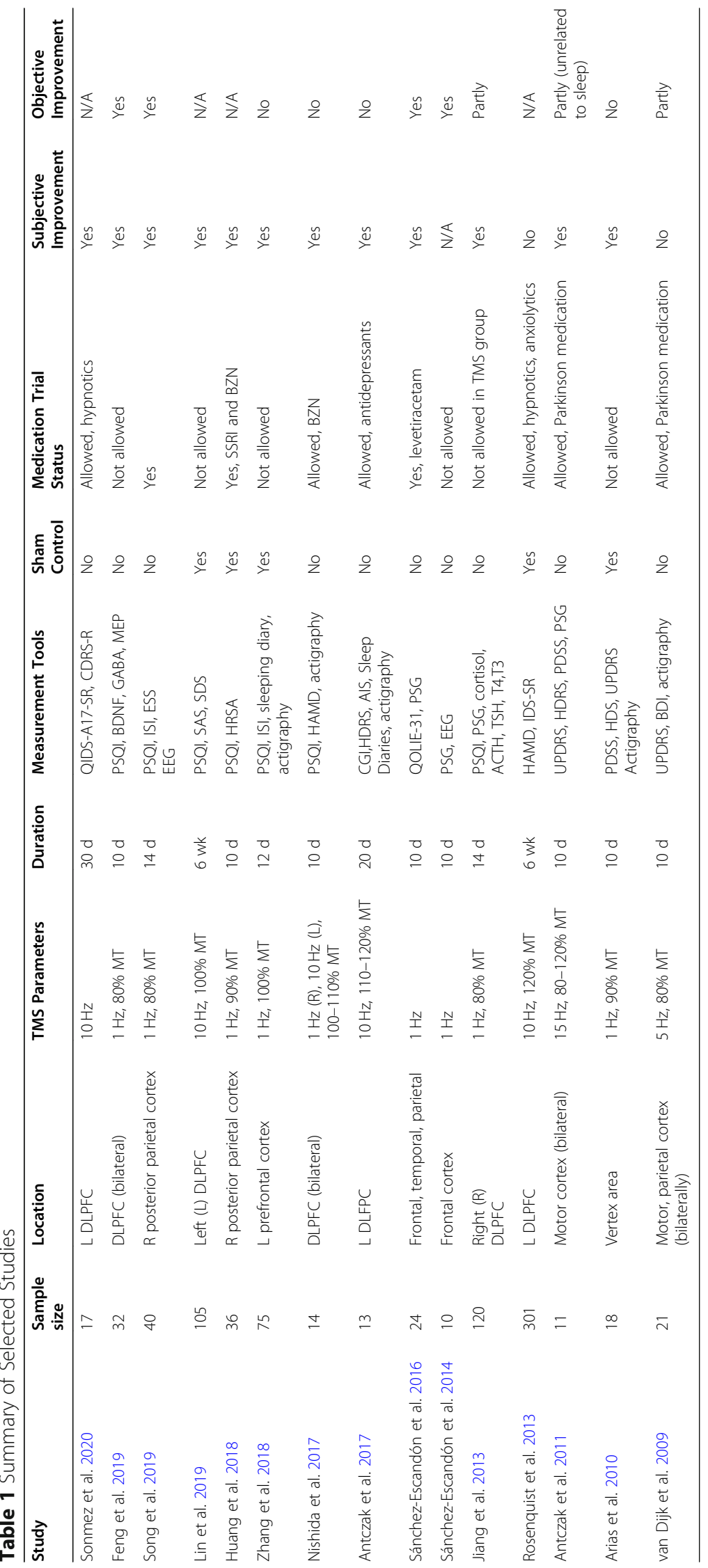


with variable results (Lefaucheur et al. 2014), it is possible that patients with primary insomnia, which is a condition known to present with cortical hyperexcitability (Lanza et al. 2015; Riemann et al. 2015), could benefit from a LF-continuous stimulus protocol thought to be inhibitory in nature (Valero-cabré et al. 2017; Chen et al. 1997; Romero et al. 2002). Interestingly, a recent study using excitatory HF rTMS in depressed adolescents found that hypersomnia symptoms improved significantly, while symptoms of insomnia did not (Sonmez et al. 2020). These findings could reflect the effect of excitatory HF rTMS on potentially underactive cortex of patients with hypersomnia, an effect potentially seen in another study (Lai et al. 2017). In contrast with individuals with insomnia, whose cortex is likely to be already overactive, HF treatment may not achieve sleep improvement. In addition, the aforementioned recent study also found preliminary evidence of a seemingly positive intrinsic effect on sleep from rTMS independent of its antidepressant effects (Sonmez et al. 2020).

However, a recent study suggests how rTMS's effect on cortical areas may be more related to functional integration of each area rather than frequency settings (Castrillon et al. 2019). In one study, $1 \mathrm{~Hz}$ rTMS was applied to patients in the frontal and occipital cortices, and the modulatory effects were different. While the occipital cortex was locally inhibited when $1 \mathrm{~Hz}$ rTMS was applied, the frontal cortex showed a paradoxical decrease in inhibition with the same $1 \mathrm{~Hz}$ stimulation. As such, this recent study highlights inconsistency in our current understanding of how rTMS frequencies work and suggests that further study is needed to investigate if there is a more complex underlying mechanism. Furthermore, occipital stimulation increased functional connectivity of local and distant areas while frontal stimulation decreased such functional connectivity.

The effects of rTMS on sleep have also been studied in Parkinson disease in two separate studies (van Dijk et al. 2009; Antczak et al. 2011), both of which applied HF over either the motor or parietal cortex. Both studies found improvement in sleep by subjective and objective measures. However, one of the studies found that the improvement seen on polysomnographic (PSG) studies was likely the result of improvement in nocturnal motor symptoms of Parkinson disease more than actual influence on sleep itself (Antczak et al. 2011). The other study found no changes in motor symptoms, but did find improvement in actigraphic recordings if rTMS was applied to the parietal cortex (van Dijk et al. 2009). These two studies used different questionnaires and measuring techniques, which could explain the conflicting results (Antczak et al. 2011). The heterogenous results of these studies on sleep illustrate that rTMS's effect is complex, due to variations in rTMS settings, target brain regions, as well as the underlying neurological conditions.

\section{Sleep measurement outcomes}

Nearly all studies showed an improvement in the subjective or self-reported sleep measurements. The Pittsburgh Sleep Quality Index (PSQI), one of the most-used questionnaires in the studies we reviewed, reported improved results either moderately or substantially in most cases. Similar results were reported by the Epworth Sleepiness Scale (ESS) and the Insomnia Severity Index (ISI). The extent to which patient bias (e.g., a psychological component of sleep problems) may have influenced these seemingly positive results is unknown, given the potential for inaccurate reporting in patient surveys. Studies also examined objective data such as that obtained from PSG and actigraphy.

Other studies examined rTMS's effect on different conditions such as epilepsy or MDD. In one epilepsy study, patients were administered LF rTMS in areas where their epileptic activity was highest, and sleep improvements were found by questionnaires and PSG parameters (Sánchez-Escandón et al. 2016). A different study focusing on rTMS in MDD and using PSQI and actigraphy had mixed findings. While PSQI questionnaires after rTMS treatment showed improvement, actigraphic results displayed no significant sleep changes (Nishida et al. 2017).

A recent systematic review examined the results of rTMS versus sham stimulation analyzing the pooled effect size of PSQI in nine selected studies (Jiang et al. 2019). rTMS was found to produce a substantial improvement in PSQI score with the highest score change in a 30-day treatment regimen. However, results observed for sham stimulation were also substantial, with $73.5 \%$ of the effect of active rTMS being produced by sham rTMS. This raises the question of whether improvement was truly due to the effect of rTMS or if placebo effect had a larger influence. It seems quite plausible that in chronic insomnia, a condition with a strong psychological component, patients may believe that their sleep will improve because of trial participation. This placebo effect could be explained by a positive feedback mechanism which has been proposed in at least one other instance (Huang et al. 2018).

One sham-controlled trial of the effect of rTMS on sleep in patients with Parkinson disease also revealed substantial placebo-related improvement on subjective questionnaires (i.e., Parkinson Disease Sleep Scale, Hamilton Depression Rating Scale, Unified Parkinson Disease Rating Scale), with sleep improvement found equally between active and sham stimulation groups (Arias et al. 2010). Interestingly, in this trial rTMS showed no changes in actigraphy parameters, which suggests that subjective measures can often yield positive results compared to objective ones.

Another sham-controlled trial with similar findings employed rTMS combined with acupuncture on chronic 
insomnia while using both subjective (PSQI, ISI, sleep diaries) and objective (actigraphy) data (Zhang et al. 2018). In this trial, improvement in subjective measures were reported in both active and sham groups, though more significantly in the active group. Similar to the previous Parkinson trial (Arias et al. 2010), objective actigraphic data in this study showed no differences between groups which again suggests how rTMS trials seem to have better subjective therapeutic results than objective ones (Zhang et al. 2018).

In contrast, a third sham-controlled study measuring the effect of rTMS in sleep and mood in patients with drug abstinence found no evidence of a placebo effect, with appreciable differences between active and sham rTMS (Lin et al. 2019). However, although this study had positive PSQI changes, it was only a mild positive effect in the rTMS group. Furthermore, it used HF rTMS on patients with symptoms of depression and anxiety due to drug abstinence and therefore it was difficult to assess how much of the sleep changes resulted from an intrinsic effect of rTMS on sleep versus the experienced mood improvement.

The fourth and last sham-controlled study we examined involved the use of LF rTMS in the parietal cortex in patients who had generalized anxiety disorder with comorbid insomnia while measuring Hamilton Rating Scale for Anxiety (HRSA), and PSQI (Huang et al. 2018). In this trial, PSQI improvements were seen in active rTMS but not in sham stimulation, making this the second example of a sham-controlled trial without pronounced placebo effect. Additionally, a positive correlation was seen between improvement in the HRSA anxiety scores and PSQI scores which could suggest that sleep improvement was associated with anxiety improvement. It was questioned whether the improvement seen in both insomnia and anxiety was independent, and whether rTMS really had an intrinsic role in sleep (Rosenquist and McCall 2019). Since anxiety disorders and insomnia often manifest together, a correlation in improvement between the two conditions could be plausible.

\section{Biological measurement outcomes}

In one study of patients with primary insomnia (Feng et al. 2019), $\gamma$-aminobutyric acid (GABA) neurotransmitter levels and brain-derived neurotrophic factor (BDNF) levels in blood serum were measured as objective indicators of sleep with higher levels associated to better sleep regulation (Datta et al. 2015; Gottesman 2002). Motorevoked potentials (MEP) were also measured to reflect cortical excitability. Analysis of results after rTMS found increased serum levels of GABA and BDNF, decreased motor evoked potentials indicative of diminished cortical excitability, and an improvement in the PSQI scale. This study found higher BDNF and GABA levels in patients with lower (improved) PSQI scores. (Feng et al. 2019)

In another study, rTMS was compared to medication and to cognitive behavioral therapy in chronic insomnia (Jiang et al. 2013). They performed assessments with PSG and PSQI, as well as measurements on cortisol, adrenocorticotrophic hormone, thyroid-stimulating hormone, and T4/T3 levels (Jiang et al. 2013). Findings showed that individuals treated with rTMS had greater improvements in PSQI and hormonal levels, when compared to the medication and cognitive behavioral therapy groups. However, based on PSG findings, rTMS only showed superior improvement in stage 3 and rapid-eye movement sleep. Overall, this study found that the largest improvement by PSG parameters was in the medication group.

In combination with PSG and questionnaires, two studies used electroencephalogram (EEG) to examine abnormalities present in patients suffering from chronic insomnia (Sánchez-Escandón et al. 2014; Song et al. 2019). EEG recordings were done before and after rTMS sessions for comparison. In one study the frequency of EEG abnormalities decreased after rTMS treatment while PSG parameters of sleep efficiency and total sleep time improved significantly (Sánchez-Escandón et al. 2014). In the other study, EEG recordings of insomnia patients showed over-active or under-active brain regions as compared to healthy controls (Song et al. 2019). After rTMS treatment, EEG data of these areas showed moderate improvement in reversing back to normal activity level. In addition, questionnaires used in this study (PSQI, ISI, ESS) also showing improved ratings.

\section{Discussion}

This review found intriguing evidence of rTMS's potential impact in sleep disorders. While a number of studies have consistently found subjective sleep improvement after rTMS, objective sleep improvement was less consistent (see Table 1 Summary of Selected Studies). Only a minority of studies were sham-controlled (see Table 1 and supplemental Table S1) to account for placebo effect. Among sham-controlled studies, some studies indeed showed a strong placebo effect of rTMS (Jiang et al. 2019; Arias et al. 2010), while placebo effect was small in other studies (Huang et al. 2018; Lin et al. 2019). Overall, studies showed variability in study characteristics and results, with most studies applying low frequency rTMS targeting the DLPFC or parietal cortex

\section{Limitations of recent studies}

There are several limitations of this research. First, studies on rTMS and sleep are scarce. After applying exclusion criteria, only a handful of studies were found. This 
is likely due to the novelty in the application of rTMS in sleep, and much more work remains to be done.

Second, the majority of studies were not shamcontrolled, likely explaining the significant placebo effect in some studies (Jiang et al. 2019; Arias et al. 2010; Zhang et al. 2018). Only 5 studies employed sham stimulation in their control groups (Huang et al. 2018; Arias et al. 2010; Zhang et al. 2018; Lin et al. 2019; Rosenquist et al. 2013).

Third, a substantial number of recent studies have small sample sizes (eight of total selected studies with fewer than 30 patients). In addition, trials should strive to control for medication usage to avoid interfering with results. Finally, studies should aim to employ some form of objective measurement to evaluate if subjective improvement is mainly the result of patient expectation.

In addition, a known occurrence in previous rTMS research is the variability of results and lack of protocol standardization. Analysis of the included studies indicate how brain area, frequency, treatment duration and medication status vary. In Supplemental Table S1, shamcontrolled studies were grouped for further comparison and demonstrate the heterogenicity of the studies.

\section{Future directions}

Recent findings of rTMS frequency and modulatory effects challenge the old paradigm of LF being inhibitory and HF being excitatory (Castrillon et al. 2019). Different brain locations may have different responses to the same rTMS frequency due to functional integration with the overall neural networks. More research is needed in this area.

In a recent study on MDD, a novel rTMS treatment paradigm with intermittent theta-burst stimulation (TBS) have yielded better results (Cole et al. 2019). TBS is a relatively recent protocol mode of rTMS in which magnetic pulses are applied in bursts with advantages of reduced time to complete TMS sessions from about $30-40 \mathrm{~min}$ to 3 min with non-inferior results (Blumberger et al. 2018; Bakker et al. 2015). This study used a novel protocol which involved multiple daily sessions, increased stimulation pulse dose, as well as guided targeting of the left DLPFC to subgenual anterior cingulate cortex circuit. This new rTMS TBS protocol resulted in $90 \%$ of patients reaching remission criteria for MDD (Cole et al. 2019).

Increasing use of TBS rTMS in MDD could provide new insights into frequency and modulatory effects on sleep (Cole et al. 2019). Indeed, previous TMS studies suggest initial nonresponders improve with further treatment sessions - with a greater and longer lasting therapeutic effects after higher total cumulative number of TMS sessions (Yip et al. 2017; Valero-Cabré et al. 2008). To our knowledge, usage of TBS rTMS in insomnia and sleep has not been reported and could be an approach worth exploring in the coming years. We theorize it could be beneficial to use continuous TBS, a mode of theta burst delivery in which trains of pulses are delivered continuously over $40 \mathrm{~s}$, and is believed to decrease cortical excitability in cortical areas for up to $50 \mathrm{~min}$ (Huang et al. 2005; Wischnewski and Schutter 2015). As cortical hyperexcitability can be a feature of primary insomnia, we theorize that continuous TBS could offer inhibitory effect and should be studied in primary insomnia in a sham-controlled trial.

Two of the main cortical areas of interest in previous studies for primary insomnia are the DLPFC (Feng et al. 2019; Jiang et al. 2013) and the parietal cortex (Huang et al. 2018; Li et al. 2014), which are good initial focus points for trial design as so far they appear to have been widely studied in TMS applied to insomnia and have had positive outcomes in previous trials as seen in this review. Nevertheless, as the science evolves in sleep medicine, other cortical areas or neuro circuits may appear over time as viable treatment targets, and more research is needed. For sleep pathologies other than primary insomnia, it is likely that different brain areas could be used, and exploring other rTMS settings (e.g., frequency) could also be an option depending on each pathology's specific pathophysiology.

Regarding optimal characteristics in future trials for insomnia, we propose the use of randomized shamcontrolled trials with both a subjective component (i.e., questionnaires) and an objective one (i.e., polysomnography or actigraphy) to clarify if perceived rTMS sleep improvements are only due to subjective patient bias. As seen previously, sham-controlled studies showed heterogenous results; with some studies displaying strong placebo effects (Jiang et al. 2019; Arias et al. 2010) while others were less pronounced (Huang et al. 2018; Lin et al. 2019). Due to these findings it will be important to use sham-stimulation in an attempt to account for any possible placebo effect. For comparison of sham-controlled studies and studies with improvement in objective measures, see supplemental Table S1 and S2, respectively. Based on our review, we found that studies which used both subjective and objective measures showed little or no objective measurement changes, while sleep improvement was more consistently reported by subjective measures (Nishida et al. 2017; Arias et al. 2010; Zhang et al. 2018; Jiang et al. 2013). This question of whether rTMS has an intrinsic positive effect on insomnia and sleep, demonstrated by objective measures, is what drives our interest for further study.

\section{Conclusion}

In conclusion, rTMS is a fast evolving field and holds great potential as treatment for various neurological and mental disorders. Our review on rTMS and insomnia found mixed results, with most studies showed subjective sleep improvements, while only a handful 
demonstrated objective sleep improvement. The presence of placebo effect in some studies makes a strong case for the need of sham-control in future trials. However, our review suggests potential applications of rTMS in insomnia, as well as, in other sleep disorders in the future. At this time, much research remain to be done to investigate optimal modalities, brain targets and therapeutic approaches in specific sleep conditions.

\section{Supplementary Information}

The online version contains supplementary material available at https://doi. org/10.1186/s41606-020-00057-9.

\section{Additional file 1: Tables S1 and S2}

\section{Abbreviations}

ACTH: Adrenocorticotropic hormone; AIS: Athens insomnia scale; BDI: Beck Depression Inventory; BDNF: Brain-derived neurotrophic factor; BZN: Benzodiazepines; CDRS-R: Children's Depression Rating Scale - Revised; CGI: Clinical Global Impression; DLPFC: Dorsolateral Prefrontal Cortex; EEG: Electroencephalogram; ESS: Epworth Sleepiness Scale; GABA: Gamma aminobutyric acid; HAMD: Hamilton Depression Scale; HDRS: Hamilton Depression Rating Scale; HDS: Hamilton Depression Scale; HRSA: Hamilton Rating Scale for Anxiety; IDS-SR: Inventory of Depressive Symptoms-Self Report; ISI: Insomnia Severity Index; MEP: Motor Evoked Potential; MT: Motor Threshold; N/A: Not applicable; PDSS: Parkinson's Disease Sleep Scale; PSG: Polysomnography; PSQI: Pittsburgh Sleep Quality Index; QIDS-A17SR: Quick Inventory of Depressive Symptomatology - Adolescent (17 Item) Self Report; QOLIE-31: Quality of Life in Epilepsy Inventory; SAS: Self-rating Anxiety Scale; SDS: Self-rating Depression Scale; SSRI: Selective serotonin reuptake inhibitor; TSH: Thyroid stimulating hormone; TMS: Transcranial Magnetic Stimulation; UPDRS: Unified Parkinson's Disease Rating Scale

\section{Acknowledgements}

We thank Carl Clingman for his artwork contribution to the figure.

\section{Authors' contributions}

$\mathrm{RO}$ conducted the literature search. RO and JC were involved in conception, analysis, and interpretation of this review. All authors were involved in the writing, revision and final approval of this article.

\section{Funding}

None.

\section{Availability of data and materials \\ Not applicable.}

\section{Ethics approval and consent to participate}

Not applicable.

\section{Consent for publication}

Not applicable.

\section{Competing interests}

$\mathrm{RO}$ and JC declared no conflict of interest. SK has received equipment support from Neuronetics, Inc. PC has received research grant support from Pfizer, Inc.; equipment support from Neuronetics, Inc:; and received supplies and genotyping services from Assurex Health, Inc. for investigator-initiated studies. He is the primary investigator for a multicenter study funded by Neuronetics, Inc. and a site primary investigator for a study funded by NeoSync, Inc. Dr. Croarkin is a consultant for Procter \& Gamble Company and Myriad Neuroscience.

\section{Author details}

${ }^{1}$ University of Navarra, Pamplona, Spain. ${ }^{2}$ Department of Psychiatry and Psychology, Mayo Clinic, Rochester, MN, USA. ${ }^{3}$ Division of Pulmonary, Allergy and Sleep Medicine, Mayo Clinic, 4500 San Pablo Rd, Jacksonville, FL 32224, USA.
Received: 13 May 2020 Accepted: 18 December 2020

Published online: 03 February 2021

\section{References}

Ahmadizadeh MJ, Rezaei M. Unilateral right and bilateral dorsolateral prefrontal cortex transcranial magnetic stimulation in treatment post-traumatic stress disorder: a randomized controlled study. Brain Res Bull. 2018;140:334-40. https://doi.org/10.1016/j.brainresbull.2018.06.001.

Aleman A. Use of repetitive transcranial magnetic stimulation for treatment in psychiatry. Clin Psychopharmacol Neurosci. 2013;11(2):53-9. https://doi.org/ 10.9758/cpn.2013.11.2.53.

Antczak J, Rakowicz M, Banach M, et al. The influence of repetitive transcranial magnetic stimulation on sleep in Parkinson's disease. Biocybern Biomed Eng. 2011;31(3):35-46. https://doi.org/10.1016/S0208-5216(11)70017-1.

Antczak JM, Poleszczyk A, Wichniak A, Rakowicz M, Parnowski TJ. The influence of the repetitive transcranial magnetic stimulation on sleep quality in depression. Psychiatr Pol. 2017;51(5):845-57. https://doi.org/10.12740/PP/ 68503. English, Polish. Epub 2017 Oct 29. PMID: 29289965.

Arias P, Vivas J, Grieve KL, Cudeiro J. Double-blind, randomized, placebo controlled trial on the effect of 10 days low-frequency rTMS over the vertex on sleep in Parkinson's disease. Sleep Med. 2010;11(8):759-65. https://doi.org/ 10.1016/j.sleep.2010.05.003.

Bakker N, Shahab S, Giacobbe P, et al. RTMS of the dorsomedial prefrontal cortex for major depression: safety, tolerability, effectiveness, and outcome predictors for $10 \mathrm{~Hz}$ versus intermittent theta-burst stimulation. Brain Stimul. 2015:8(2):208-15. https://doi.org/10.1016/j.brs.2014.11.002.

Berlim MT, Neufeld NH, Van den Eynde F. Repetitive transcranial magnetic stimulation (rTMS) for obsessive-compulsive disorder (OCD): an exploratory meta-analysis of randomized and sham-controlled trials. J Psychiatr Res. 2013b;47(8):999-1006. https://doi.org/10.1016/j.jpsychires. 2013.03.022.

Berlim MT, Van Den Eynde F, Jeff Daskalakis Z. Clinically meaningful efficacy and acceptability of low-frequency repetitive transcranial magnetic stimulation (rTMS) for treating primary major depression: a meta-analysis of randomized, double-blind and sham-controlled trials. Neuropsychopharmacology. 2013a; 38(4):543-51. https://doi.org/10.1038/npp.2012.237.

Berlim MT, Van Den Eynde F, Tovar-Perdomo S, Daskalakis ZJ. Response, remission and drop-out rates following high-frequency repetitive transcranial magnetic stimulation (rTMS) for treating major depression: a systematic review and meta-analysis of randomized, double-blind and sham-controlled trials. Psychol Med. 2014:44(2):225-39. https://doi.org/10.1017/ S0033291713000512

Blumberger DM, Vila-Rodriguez F, Thorpe KE, et al. Effectiveness of theta burst versus high-frequency repetitive transcranial magnetic stimulation in patients with depression (THREE-D): a randomised non-inferiority trial. Lancet. 2018; 391(10131):1683-92. https://doi.org/10.1016/S0140-6736(18)30295-2.

Boyer L, Dousset A, Roussel P, et al. rTMS in fibromyalgia: a randomized trial evaluating QoL and its brain metabolic substrate. Neurology. 2014;82(14): 1231-8. https://doi.org/10.1212/WNL.0000000000000280.

Carmi L, Alyagon U, Barnea-Ygael N, Zohar J, Dar R, Zangen A. Clinical and electrophysiological outcomes of deep TMS over the medial prefrontal and anterior cingulate cortices in OCD patients. Brain Stimul. 2018;11(1):158-65. https://doi.org/10.1016/j.brs.2017.09.004.

Carmi L, Tendler A, Bystritsky A, et al. Efficacy and safety of deep transcranial magnetic stimulation for obsessive-compulsive disorder: a prospective multicenter randomized double-blind placebo-controlled trial. Am J Psychiatry. 2019;176(11):931-8. https://doi.org/10.1176/appi.ajp.2019. 18101180.

Castrillon G, Sollmann N, Kurcyus K, Razi A, Krieg SM, Riedl V. The physiological effects of non-invasive brain stimulation fundamentally differ across the human cortex. bioRxiv. 2019:639237. https://doi.org/10.1101/639237.

Chen R, Classen J, Gerloff C, et al. Depression of motor cortex excitability by lowfrequency transcranial magnetic stimulation. Neurology. 1997:48(5):1398-403. https://doi.org/10.1212/WNL.48.5.1398

Cirillo P, Gold AK, Nardi AE, et al. Transcranial magnetic stimulation in anxiety and trauma-related disorders: a systematic review and meta-analysis. Brain Behav. 2019:9(6):1-17. https://doi.org/10.1002/brb3.1284.

Civardi C, Boccagni C, Vicentini R, et al. Cortical excitability and sleep deprivation: a transcranial magnetic stimulation study. J Neurol Neurosurg Psychiatry. 2001;71(6):809-12. https://doi.org/10.1136/jnnp.71.6.809. 
Cole E, Gulser M, Stimpson K, et al. Stanford accelerated intelligent neuromodulation therapy for treatment-resistant depression (SAINT-TRD). Brain Stimul. 2019;12(2):402. https://doi.org/10.1016/j.brs.2018.12.299.

Datta S, Knapp CM, Koul-tiwari R, Drive C. The Homeostatic Regulation of REM Sleep: A role for Localized Expression of Brain-Derived Neurotrophic Factor in the Brainstem. Behav Brain Res. 2015;292:381-92. https://doi.org/10.1016/j. bbr.2015.06.038.

Feng J, Zhang Q, Zhang C, Wen Z, Zhou X. The effect of sequential bilateral lowfrequency rTMS over dorsolateral prefrontal cortex on serum level of BDNF and GABA in patients with primary insomnia. Brain Behav. 2019;9(2):1-8. https://doi.org/10.1002/brb3.1206.

Fitzgerald PB, Hoy KE, Herring SE, et al. A double blind randomized trial of unilateral left and bilateral prefrontal cortex transcranial magnetic stimulation in treatment resistant major depression. J Affect Disord. 2012;139(2):193-8. https://doi.org/10.1016/j.jad.2012.02.017.

George MS, Lisanby SH, Avery D, et al. Daily left prefrontal transcranial magnetic stimulation therapy for major depressive disorder: a sham-controlled randomized trial. Arch Gen Psychiatry. 2010;67(5):507-16. https://doi.org/10. 1001/archgenpsychiatry.2010.46.

Gottesman. GABA mechanisms and sleep. Neuroscience. 2002;111(2):231-9. https://doi.org/10.1016/s0306-4522(02)00034-9.

Hallett M. Transcranial magnetic stimulation and the human brain. Nature. 2000 406(6792):147-50. https://doi.org/10.1038/35018000. PMID: 10910346.

Huang YZ, Edwards MJ, Rounis E, Bhatia KP, Rothwell JC. Theta burst stimulation of the human motor cortex. Neuron. 2005;45(2):201-6. https://doi.org/10. 1016/j.neuron.2004.12.033

Huang Z, Li Y, Bianchi MT, et al. Repetitive transcranial magnetic stimulation of the right parietal cortex for comorbid generalized anxiety disorder and insomnia: a randomized, double-blind, sham-controlled pilot study. Brain Stimul. 2018;11(5):1103-9. https://doi.org/10.1016/j.brs.2018.05.016.

Huber R, Esser SK, Ferrarelli F, Massimini M, Peterson MJ, Tononi G. TMS-induced cortical potentiation during wakefulness locally increases slow wave activity during sleep. PLoS One. 2007;2(3):1-7. https://doi.org/10.1371/journal.pone. 0000276.

Jiang B, He D, Guo Z, Mu Q, Zhang L. Efficacy and placebo response of repetitive transcranial magnetic stimulation for primary insomnia. Sleep Med. 2019;63: 9-13. https://doi.org/10.1016/j.sleep.2019.05.008.

Jiang C-g, Zhang T, Yue F-g, Yi M-I, Gao D. Efficacy of repetitive Transcranial magnetic stimulation in the treatment of patients with chronic primary insomnia. Cell Biochem Biophys. 2013;67(1):169-73. https://doi.org/10.1007/ s12013-013-9529-4

Karsen EF, Watts BV, Holtzheimer PE. Review of the effectiveness of transcranial magnetic stimulation for post-traumatic stress disorder. Brain Stimul. 2014; 7(2):151-7. https://doi.org/10.1016/j.brs.2013.10.006.

Kimbrell TA, Ketter TA, George MS, et al. Regional cerebral glucose utilization in patients with a range of severities of unipolar depression. Biol Psychiatry. 2002;51(3):237-52. https://doi.org/10.1016/S0006-3223(01)01216-1.

Kozel FA, Motes MA, Didehbani N, et al. Repetitive TMS to augment cognitive processing therapy in combat veterans of recent conflicts with PTSD: a randomized clinical trial. J Affect Disord. 2018;229:506-14. https://doi.org/10. 1016/j.jad.2017.12.046.

Kreuzer P, Langguth B, Popp R, et al. Reduced intra-cortical inhibition after sleep deprivation: a transcranial magnetic stimulation study. Neurosci Lett. 2011; 493(3):63-6. https://doi.org/10.1016/j.neulet.2011.02.044.

Lai JB, Han MM, Xu Y, Hu SH. Effective treatment of narcolepsy-like symptoms with high-frequency repetitive transcranial magnetic stimulation: a case report. Medicine (United States). 2017;96(46):11-3. https://doi.org/10.1097/ MD.0000000000008645.

Lanza G, Cantone M, Lanuzza B, et al. Distinctive patterns of cortical excitability to transcranial magnetic stimulation in obstructive sleep apnea syndrome, restless legs syndrome, insomnia, and sleep deprivation. Sleep Med Rev. 2015;19:39-50. https://doi.org/10.1016/j.smrv.2014.04.001.

Lefaucheur JP, André-Obadia N, Antal A, et al. Evidence-based guidelines on the therapeutic use of repetitive transcranial magnetic stimulation (rTMS). Clin Neurophysiol. 2014;125(11):2150-206. https://doi.org/10.1016/j.clinph.2014.05.021.

Li Y, Wang E, Zhang H, et al. Functional connectivity changes between parietal and prefrontal cortices in primary insomnia patients: evidence from resting-state fMRI. Eur J Med Res. 2014;19(1):1-7. https://doi.org/10.1186/2047-783X-19-32.

Lin J, Liu X, Li H, et al. Chronic repetitive transcranial magnetic stimulation (rTMS) on sleeping quality and mood status in drug dependent male inpatients during abstinence. Sleep Med. 2019;58:7-12. https://doi.org/10.1016/j.sleep.2019.01.052.
Lin YC, Feng Y, Zhan SQ, et al. Repetitive transcranial magnetic stimulation for the treatment of restless legs syndrome. Chin Med J. 2015;128(13):1728-31. https://doi.org/10.4103/0366-6999.159344.

Luber B, Steffener J, Tucker A, et al. Extended remediation of sleep deprivedinduced working memory deficits using fMRI-guided Transcranial magnetic stimulation. Sleep. 2013;36(6):857-71. https://doi.org/10.5665/sleep.2712.

Mayberg HS. Positron emission tomography imaging in depression: a neural systems perspective. Neuroimaging Clin N Am. 2003;13(4):805-15. https://doi. org/10.1016/S1052-5149(03)00104-7.

Mhalla A, Baudic S, De Andrade DC, et al. Long-term maintenance of the analgesic effects of transcranial magnetic stimulation in fibromyalgia. Pain. 2011;152(7):1478-85. https://doi.org/10.1016/j.pain.2011.01.034.

Murphy MJ, Peterson MJ. Sleep disturbances in depression. Sleep Med Clin. 2015; 10(1):17-23. https://doi.org/10.1016/j.jsmc.2014.11.009.

Nardone R, Höller Y, Brigo F, Tezzon F, Golaszewski S, Trinka E. Transcranial magnetic stimulation and sleep disorders: pathophysiologic insights. Sleep Med. 2013;14(11):1047-58. https://doi.org/10.1016/j.sleep.2013.04.025.

Nishida M, Kikuchi S, Nisijima K, Suda S. Actigraphy in patients with major depressive disorder undergoing repetitive transcranial magnetic stimulation an open label pilot study. J ECT. 2017;33(1):36-42. https://doi.org/10.1097/ YCT.0000000000000352

O'Reardon JP, Solvason HB, Janicak PG, et al. Efficacy and safety of Transcranial magnetic stimulation in the acute treatment of major depression: a multisite randomized controlled trial. Biol Psychiatry. 2007;62(11):1208-16. https://doi. org/10.1016/j.biopsych.2007.01.018.

Ohayon MM. Epidemiological Overview of sleep Disorders in the General Population. Sleep Med Res. 2011;2(1):1-9. https://doi.org/10.17241/smr.2011.2.1.1.

Passard A, Attal N, Benadhira R, et al. Effects of unilateral repetitive transcranial magnetic stimulation of the motor cortex on chronic widespread pain in fibromyalgia. Brain. 2007;130(10):2661-70. https://doi.org/10.1093/brain/ awm189.

Ray S, Nizamie SH, Akhtar S, Praharaj SK, Mishra BR, Zia-Ul-Haq M. Efficacy of adjunctive high frequency repetitive transcranial magnetic stimulation of left prefrontal cortex in depression: a randomized sham controlled study. J Affect Disord. 2011;128(1-2):153-9. https://doi.org/10.1016/j.jad.2010.06.027.

Riemann D, Nissen C, Palagini L, Otte A, Perlis ML, Spiegelhalder K. The neurobiology, investigation, and treatment of chronic insomnia. Lancet Neurol. 2015;14(5):547-58. https://doi.org/10.1016/S1474-4422(15)00021-6.

Riemann D, Spiegelhalder K, Feige B, et al. The hyperarousal model of insomnia: a review of the concept and its evidence. Sleep Med Rev. 2010;14(1):19-31. https://doi.org/10.1016/j.smrv.2009.04.002.

Rodrigues PA, Zaninotto AL, Neville IS, et al. Transcranial magnetic stimulation for the treatment of anxiety disorder. Neuropsychiatr Dis Treat. 2019;15:2743-61. https://doi.org/10.2147/ndt.s201407.

Romero JR, Anschel D, Sparing R, Gangitano M, Pascual-Leone A. Subthreshold low frequency repetitive transcranial magnetic stimulation selectively decreases facilitation in the motor cortex. Clin Neurophysiol. 2002;113(1):1017. https://doi.org/10.1016/S1388-2457(01)00693-9.

Rosenquist PB, Krystal A, Heart KL, Demitrack MA, McCall WV. Left dorsolateral prefrontal transcranial magnetic stimulation (TMS): sleep factor changes during treatment in patients with pharmacoresistant major depressive disorder. Psychiatry Res. 2013;205(1-2):67-73. https://doi.org/10.1016/j. psychres.2012.09.011.

Rosenquist PB, McCall WV. Does rTMS treat insomnia? Brain Stimul. 2019;12(3): 809. https://doi.org/10.1016/j.brs.2019.02.004.

Rossi S, Hallett M, Rossini PM, et al. Safety, ethical considerations, and application guidelines for the use of transcranial magnetic stimulation in clinical practice and research. Clin Neurophysiol. 2009;120(12):2008-39. https://doi.org/10. 1016/j.clinph.2009.08.016.

Sánchez-Escandón O, Arana-Lechuga Y, Terán-Pérez G, et al. Transcraneal magnetic stimulation improves sleep parameters in patients affected with Imsomnia associated to electroencephalographic abnormalities. Neurosci Med. 2014;05(01):72-7. https://doi.org/10.4236/nm.2014.51010.

Sánchez-Escandón O, Arana-Lechuga Y, Terán-Pérez G, et al. Effect of lowfrequency repetitive transcranial magnetic stimulation on sleep pattern and quality of life in patients with focal epilepsy. Sleep Med. 2016;20:37-40. https://doi.org/10.1016/j.sleep.2015.11.022.

Song P, Lin H, Li S, et al. Repetitive transcranial magnetic stimulation (rTMS) modulates time-varying electroencephalography (EEG) network in primary insomnia patients: a TMS-EEG study. Sleep Med. 2019;56:157-63. https://doi. org/10.1016/j.sleep.2019.01.007. 
Sonmez Al, Kucuker MU, Lewis CP, et al. Improvement in hypersomnia with high frequency repetitive transcranial magnetic stimulation in depressed adolescents: preliminary evidence from an open-label study. Prog NeuroPsychopharmacology Biol Psychiatry. 2020;97:109763. https://doi.org/10.1016/ j.pnpbp.2019.109763.

Valero-cabré A, Amengual JL, Stengel C, Pascual-leone A, Coubard OA Neuroscience and biobehavioral reviews Transcranial magnetic stimulation in basic and clinical neuroscience : a comprehensive review of fundamental principles and novel insights. Neurosci Biobehav Rev. 2017;83(October):381404. https://doi.org/10.1016/j.neubiorev.2017.10.006.

Valero-Cabré A, Pascual-Leone A, Rushmore RJ. Cumulative sessions of repetitive transcranial magnetic stimulation (rTMS) build up facilitation to subsequent TMS-mediated behavioural disruptions. Eur J Neurosci. 2008;27(3):765-74. https://doi.org/10.1111/j.1460-9568.2008.06045.x.

Van Der Werf YD, Altena E, Van Dijk KD, et al. Is disturbed intracortical excitability a stable trait of chronic insomnia? A study using transcranial magnetic stimulation before and after multimodal sleep therapy. Biol Psychiatry. 2010; 68(10):950-5. https://doi.org/10.1016/j.biopsych.2010.06.028.

van Dijk KD, Møst EIS, van Someren EJW, Berendse HW, van der Werf YD. Beneficial effect of transcranial magnetic stimulation on sleep in Parkinson's disease. Mov Disord. 2009;24(6):878-84. https://doi.org/10.1002/mds.22462.

Watts BV, Landon B, Groft A, Young-Xu Y. A sham controlled study of repetitive transcranial magnetic stimulation for posttraumatic stress disorder. Brain Stimul. 2012;5(1):38-43. https://doi.org/10.1016/j.brs.2011.02.002.

Wischnewski M, Schutter DJLG. Efficacy and time course of theta burst stimulation in healthy humans. Brain Stimul. 2015;8(4):685-92. https://doi.org/ 10.1016/j.brs.2015.03.004.

Yip AG, George MS, Tendler A, Roth Y, Zangen A, Carpenter LL. 61\% of unmedicated treatment resistant depression patients who did not respond to acute TMS treatment responded after four weeks of twice weekly deep TMS in the Brainsway pivotal trial. Brain Stimul. 2017;10(4):847-9. https://doi. org/10.1016/j.brs.2017.02.013.

Zhang Y, Liao W-J, Xia W. Effect of acupuncture cooperated with low-frequency repetitive Transcranial magnetic stimulation on chronic insomnia: a randomized clinical trial. Curr Med Sci. 2018;38(3):491-8. https://doi.org/10. 1007/s11596-018-1905-2.

Zhou WN, Fu HY, Du YF, et al. Short-term effects of repetitive transcranial magnetic stimulation on sleep bruxism-a pilot study. Int J Oral Sci. 2016;8(1): 61-5. https://doi.org/10.1038/ijos.2015.35.

\section{Publisher's Note}

Springer Nature remains neutral with regard to jurisdictional claims in published maps and institutional affiliations.

Ready to submit your research? Choose BMC and benefit from:

- fast, convenient online submission

- thorough peer review by experienced researchers in your field

- rapid publication on acceptance

- support for research data, including large and complex data types

- gold Open Access which fosters wider collaboration and increased citations

- maximum visibility for your research: over $100 \mathrm{M}$ website views per year

At $\mathrm{BMC}$, research is always in progress.

Learn more biomedcentral.com/submissions 